\title{
Relativistic Treatment of Quantum Mechanical Gravitational-Harmonic Oscillator Potential
}

\author{
E. P. Inyang, B. I. Ita and E. P. Inyang
}

\section{ABSTRACT}

\begin{abstract}
In this work, we solved the Klein-Gordon equation with quantum mechanical gravitational plus harmonic oscillator potential via the parametric Nikiforov-Uvarov method. The energy equation and the corresponding un-normalized wave function in terms of Laguerre polynomials were obtained. The numerical values for the $S$-wave was obtained.
\end{abstract}

Keywords: Klein-Gordon equation; Quantum mechanical gravitational potential; Harmonic oscillator potential; Nikiforov-Uvarov method
Published Online: June 24, 2021

ISSN: $2684-4451$

DOI :10.24018/ejphysics.2021.3.3.83

\section{E. P. Inyang}

Theoretical Physics Group, Department of Physics, University of Calabar, P.M.B 1115, Calabar Nigeria.

(e-mail: inyang.ephraim@ ${ }^{\circledR}$ gmail.com)

B. I. Ita

Physical and Theoretical Chemistry

Group, Department of Pure and Applied

Chemistry, University of Calabar,

Calabar, Nigeria.

(e-mail: iserom2001 @ yahoo.com)

E. P. Inyang*

Department of Pure and Applied Sciences, National Open University of Nigeria, Abuja

Theoretical Physics Group, Department of Physics, University of Calabar, P.M.B 1115, Calabar, Nigeria.

(e-mail: etidophysics@gmail.com)

*Corresponding Author

The purpose of the present work is to present the solutions of the KGE with the quantum mechanical gravitational potential (QMGP) plus harmonic oscillator potential (HOP) of the form [33] and obtain the S-wave.

$V(r)=m g r+\delta e^{-k r}+\frac{1}{2} \mu \omega^{2} r^{2}$

where $r$ is the displacement, $k$ is momentum, $m$ is the mass, $g$ is gravitational acceleration, $\delta$ is an adjustable parameter, $\mu$ is the reduced mass and $\omega$ is the angular frequency. The QMGP could be used to calculate the energy of a body falling under gravity from quantum mechanical point of view. Berberan-Santos et al [34] have studied the motion of a particle in a gravitational field using the QMGP without the exponential term. They obtained the classical and quantum mechanical position probability distribution function for the particle. The HOP has been widely studied in the literature. For example, Amore and Fernandez [35] studied the two-particle harmonic oscillator in a one dimensional box and obtained energy eigen values which were comparable with energies from variational and perturbation methods. Jasim et al [36] also investigated the single particle level density in a harmonic - oscillator potential well and obtained very interesting results. They 
studied the properties of the partial level density and the total level density numerically for the harmonic oscillator potential well. Also, Ikot et al [37] derived the energy eigenvalues and the corresponding eigen functions for the two - dimensional harmonic oscillator potential in cartesian and polar coordinates using the Nikiforov -Uvarov method. Xue-Hong et al [38] determined the virial theorem for a class of quantum nonlinear harmonic oscillators.

\section{THE NIKIFOROV-UVAROV METHOD}

The Nikiforov-Uvarov (NU) method is based on the solutions of a generalized second-order linear differential equation with special orthogonal functions [39]. The Schrodinger equation of the type as

$\psi^{\prime \prime}(r)+[E-V(r)] \psi(r)=0$

can be solved by this method. This can be done by transforming (2) into an equation of hypergeometric type with appropriate coordinate transformation $s=s(r)$ to get:

$\psi^{\prime \prime}(s)+\frac{\bar{\tau}(s)}{\sigma(s)} \psi^{\prime}(s)+\frac{\bar{\sigma}(s)}{\sigma^{2}(s)} \psi(s)=0$

To find the exact solution to Eq. (3), we write $\psi(s)$ as"

$\psi(s)=\phi(s) \chi(s)$

Substitution of (4) into (3) yields (5) of hypergeometric type as:

$\sigma(s) \chi^{\prime \prime}(s)+\tau(s) \chi^{\prime}(s)+\lambda \chi(s)=0$

In (4), the wave function $\phi(s)$ is defined as the logarithmic derivative [41]:

$\frac{\phi^{\prime}(s)}{\phi(s)}=\frac{\pi(s)}{\sigma(s)}$

with $\pi(s)$ being at most first order polynomials. Also, the hypergeometric-type functions in (5) for a fixed integer $n$ is given by the Rodrigue relation as:

$\chi_{n}(s)=\frac{B_{n}}{\rho_{n}} \frac{d^{n}}{d s^{n}}\left[\sigma^{n}(s) \rho(s)\right]$

where $B_{n}$ is the normalization constant and the weight function $\rho(s)$ must satisfy the condition:

$\frac{d}{d s}\left[\sigma^{n}(s) \rho(s)\right]=\tau(s) \rho(s)$

With

$\tau(s)=\bar{\tau}(s)+2 \pi(s)$

In order to accomplish the condition imposed on the weight function $\rho(s)$ it is necessary that the polynomial $\tau(s)$ be equal to zero at some point of an interval $(a, b)$ and its derivative at this interval at $\tau^{\prime}(s)<0$ will be negative [42]. That is: $\frac{d \tau(s)}{d s}<0$

The function $\pi(s)$ and the parameter $\lambda$ required for the NU method are then defined as [43]:

$\pi(s)=\frac{\sigma^{\prime}-\bar{\tau}}{2} \pm \sqrt{\left(\frac{\sigma^{\prime}-\bar{\tau}}{2}\right)^{2}-\bar{\sigma}+k \sigma}$

$\lambda=k+\pi^{\prime}(s)$

The $s$-values in (11) are possible to evaluate if the expression under the square root be square of polynomials. This is possible if and only if its discriminant is zero. Therefore, the new eigenvalues equation becomes:

$\lambda=\lambda_{n}=-n \frac{d \tau}{d s}-\frac{n(n-1)}{2} \frac{d^{2} \sigma}{d s^{2}}, n=0,1,2, \ldots$

A comparison between (12) and (13) yields the energy eigenvalues.

Secondly, the parametric generalization of the NU method is expressed by the generalized hypergeometric-type equation:

$\psi^{\prime \prime}(s)+\frac{\left(c_{1}-c_{2} s\right)}{s\left(1-c_{3} s\right)} \psi^{\prime}(s)+\frac{1}{s^{2}\left(1-c_{3} s\right)^{2}}\left[-\xi_{1} s^{2}+\xi_{2} s-\right.$
$\left.\xi_{3}\right] \psi(s)=0$

Equation (14) is solved by comparing it with (3) and the following polynomials are obtained:

$$
\begin{aligned}
& \bar{\tau}=\left(c_{1}-c_{2} s\right), \sigma(s)=s\left(1-c_{3} s\right), \bar{\sigma}(s)=-\xi_{1} s^{2}+\xi_{2} s- \\
& \xi_{3}
\end{aligned}
$$

Now, substituting (15) into (11) gives:

$\pi(s)=c_{4}+c_{5} s \pm\left[\left(c_{6}-c_{3} k_{ \pm}\right) s^{2}+\left(c_{7}+k_{ \pm}\right) s+c_{8}\right]^{1 / 2}$

where

$c_{4}=\frac{1}{2}\left(1-c_{1}\right), c_{5}=\frac{1}{2}\left(c_{2}-2 c_{3}\right), c_{6}=c_{5}{ }^{2}+\xi_{1}, c_{7}=$

$2 c_{4} c_{5}-\xi_{2}, c_{8}=c_{4}^{2}+\xi_{3}$

The resulting value of $k$ in (16) is obtained from the condition that the function under the square-root should be square of a polynomial and we get:

$k_{ \pm}=-\left(c_{7}+2 c_{3} c_{8}\right) \pm 2 \sqrt{c_{8} c_{9}}$

where

$c_{9}=c_{3} c_{7}+c_{2}{ }^{2} c_{8}+c_{6}$

The new $\pi(s)$ for $k$ becomes:

$\pi(s)=c_{4}+c_{5} s \mp\left[\begin{array}{c}\left(\sqrt{c_{9}}+c_{3} \sqrt{c_{8}}\right) s \\ -\sqrt{c_{8}}\end{array}\right]$

$k_{\text {_value becomes }}$ 
$k_{-}=-\left(c_{7}+2 c_{3} c_{8}\right)-2 \sqrt{c_{8} c_{9}}$

Using (9), we obtain:

$\tau(s)=c_{1}+2 c_{4}-\left(c_{2}-2 c_{5}\right) s-2\left[\begin{array}{c}\left(\sqrt{c_{9}}+c_{3} \sqrt{c_{8}}\right) s \\ -\sqrt{c_{8}}\end{array}\right]$

The physical condition for the bound state solution is $\tau^{\prime}<$ 0 and thus:

$\tau^{\prime}(s)=-2 c_{3}-2\left(\sqrt{c_{9}}+c_{3} \sqrt{c_{8}}\right)<0$

With the aid of (12) and (13), we obtain the energy equation as:

$\left(c_{2}-c_{3}\right) n+c_{3} n^{2}-(2 n+1) c_{5}+(2 n+1) \times$

$\times\left(\sqrt{c_{9}}+c_{3} \sqrt{c_{8}}\right)+c_{7}+2 c_{3} c_{8}+2 \sqrt{c_{8} c_{9}}=0$

The weight function $\rho(s)$ is obtained from (8) as:

$\rho(s)=s^{c_{10}-1}\left(1-c_{3} s\right)^{\frac{c_{11}}{c_{3}}-c_{10}-1}$

And together with (7), we have:

$\chi_{n}(s)=P_{n}{ }^{\left(c_{10}-1, \frac{c_{11}}{c_{3}}-c_{10}-1\right)}\left(1-2 c_{3} s\right)$

Where

$c_{10}=c_{1}+2 c_{4}+2 \sqrt{c_{8}}, c_{11}=c_{2}-2 c_{5}+2\left(\sqrt{c_{9}}+c_{3} \sqrt{c_{8}}\right)(27)$

$P_{n}^{(\alpha, \beta)}$ are the Jacobi polynomials. The second part of the wave function is obtained from (6) as:

$$
\phi(s)=s^{c_{12}}\left(1-c_{3} s\right)^{-c_{12}-\frac{c_{13}}{c_{3}}}
$$

where

$$
c_{12}=c_{4}+\sqrt{c_{8}}, c_{13}=c_{5}-\left(\sqrt{c_{9}}+c_{3} \sqrt{c_{8}}\right)
$$

Thus, the total wave function becomes:

$$
\begin{aligned}
& \psi(s)=N_{n} s^{c_{12}}\left(1-c_{3} s\right)^{-c_{12}-\frac{c_{13}}{c_{3}}} P_{n}^{\left(c_{10}-1, \frac{c_{11}}{c_{3}}-c_{10}-1\right)}(1- \\
& \left.2 c_{3} s\right)
\end{aligned}
$$

where $N_{n}$ is the normalization constant.

\section{THE KLEIN-GORDON EQUATION}

The three-dimensional $\mathrm{KG}$ equation with mixed vector and scalar potentials can be written as [30]:

$\left[\nabla^{2}+(V(r)-E)^{2}-(S(r)+M)^{2}\right] \psi(r, \theta, \phi)=0$

where $M$ is the rest mass, $E$ is the relativistic energy, and $S(r)$ and $V(r)$ are the scalar and vector potentials, respectively. $\nabla^{2}$ is the Laplacian operator, $c$ is the speed of light, and $\hbar$ is the reduced Planck's constant which have been set to unity. In spherical coordinates, the $\mathrm{KG}$ equation with the potential $V(r)$ is given as [30]:

$\left[\begin{array}{l}\frac{1}{r^{2}} \frac{\partial}{\partial r}\left(r^{2} \frac{\partial}{\partial r}\right)+\frac{1}{r^{2} \sin \theta} \frac{\partial}{\partial \theta}\left(\sin \theta \frac{\partial}{\partial \theta}\right)+\frac{1}{r^{2} \sin ^{2} \theta} \frac{\partial^{2}}{\partial \phi^{2}} \\ -2(E V(r)+M S(r))+V^{2}(r)-S^{2}(r)+E^{2}-M^{2}\end{array}\right] \psi(r, \theta, \phi)=$
$=0$

Using the common ansatz for the wave function:

$\psi(r, \theta, \phi)=\frac{R(r)}{r} Y_{l m}(\theta, \phi)$

From (32) we get the following set of equations:

$\frac{d^{2} R(r)}{d r^{2}}+\left[E^{2}-M^{2}-2(E V(r)+M S(r))+V^{2}(r)-\right.$

$\left.-S^{2}(r)-\frac{\lambda}{r^{2}}\right] R(r)=0$

$\frac{d^{2} \Theta(\theta)}{d \theta^{2}}+\cot \theta \frac{d \Theta(\theta)}{d \theta}\left(\lambda-\frac{m^{2}}{\sin ^{2} \theta}\right) \Theta(\theta)=0$

$\frac{d^{2} \Phi(\phi)}{d \phi^{2}}+m^{2} \Phi(\phi)=0$

where $\lambda=l(l+1)$ and $m^{2}$ are the separation constants. $Y_{l m}(\theta, \phi)=\Theta_{m l}(\theta) \Phi_{m}(\phi)$ is the solution of (35) and (36) and their solutions are well known as spherical harmonic functions [30]. Equation (34) is the radial part of the $\mathrm{KG}$ equation which we are interested in solving. The potential in equation (1) can be written as:

$V(r)=\beta r+V_{0} e^{-\alpha r}+\frac{1}{2} \mu \omega^{2} r^{2}$

where $\beta=m g, \alpha=k, z=r, \delta=V_{0}$. We can also write (37) as:

$V(r)=\beta r+V_{0}\left(1-\alpha r+\alpha^{2} r^{2}\right)+\frac{1}{2} \mu \omega^{2} r^{2}$

On arranging (38) we get our working potential amenable to parametric NU method as:

$V(r)=V_{0}+\left(\beta-\alpha V_{0}\right) r+\left(\alpha^{2} V_{0}+\frac{1}{2} \mu \omega^{2}\right) r^{2}$

The potential of (39) can be used to solve various quantum mechanical equations including, the Schrodinger equation (SE), Klein-Gordon equation (KGE) and Dirac equation using the NU method for their exact solutions.

\section{SOlUtions OF THE RADIAL EQUATION}

Writing (34) for $S$-waves and for a special case $V(r)=$ $S(r)$ as:

$\frac{d^{2} R(r)}{d r^{2}}+\left[E^{2}-M^{2}-2(E+M) V(r)\right] R(r)=0$

And using the potential of (39) in (40) yields: 
$\frac{d^{2} R(r)}{d r^{2}}+\left[E^{2}-M^{2}-2(E+M) V_{0}-2(E+M)(\beta-\right.$

$\left.\left.\alpha V_{0}\right) r-2(E+M)\left(\alpha^{2} V_{0}+\frac{1}{2} \mu \omega^{2}\right) r^{2}\right] R(r) 0$

Comparing (41) with (14) yields the following parameters:

$c_{1}=c_{2}=c_{3}=0, \xi_{1}=2(E+M)\left(\alpha^{2} V_{0}+\frac{1}{2} \mu \omega^{2}\right), \xi_{2}=-2(E+$ $M)\left(\beta-\alpha V_{0}\right), \xi_{3}=-\left(E^{2}-M^{2}\right)+2(E+M) V_{0}$

Other coefficients are determined as:

$c_{4}=\frac{1}{2}, c_{5}=0, c_{6}=\xi_{1}, c_{7}=-\xi_{2}, c_{8}=\frac{1}{4}+\xi_{3}, c_{9}=$

$\xi_{1}, c_{10}=1+2 \sqrt{\frac{1}{4}+\xi_{3}}, c_{11}=2 \sqrt{\xi_{1}}, c_{12}=\frac{1}{2}+$

$\sqrt{\frac{1}{4}+\xi_{3}}, c_{13}=-\sqrt{\xi_{1}}$

From (16):

$\pi(s)=\frac{1}{2} \pm\left[\xi_{1} s^{2}+\left(-\xi_{2}+k_{ \pm}\right) s+\frac{1}{4}+\xi_{3}\right]^{1 / 2}$

From (18):

$k_{ \pm}=\xi_{2} \pm 2 \sqrt{\left(\frac{1}{4}+\xi_{3}\right) \xi_{1}}$

From (22):

$\tau(s)=1-\left(2 \sqrt{\xi_{1}}\right) s-\sqrt{\frac{1}{4}+\xi_{3}}$

The negative derivative of (46) then becomes:

$\tau^{\prime}(s)=-\left(2 \sqrt{\xi_{1}}\right)<0$

The new $\pi(s)$ for the NU method is chosen as:

$\pi(s)=\frac{1}{2}-\left(\sqrt{\xi_{1}}\right) s-\sqrt{\frac{1}{4}+\xi_{3}}$

For

$k_{-}=\xi_{2}-2 \sqrt{\left(\frac{1}{4}+\xi_{3}\right) \xi_{1}}$

Now using (24), (42) and (43) we obtain the relativistic limit energy spectrum of the QMGP + HOP as:

$M^{2}-E^{2}=-\left[X-\left(\frac{E+M}{2}\right) \mu \omega^{2}\right] / Y$

where

$X=\left\{(2 n+1)\left[2(E+M)\left(\alpha^{2} V_{0}+\frac{1}{2} \mu \omega^{2}\right)+2(E+\right.\right.$

M) $\left.\left.\left(\beta-\alpha V_{0}\right)\right]\right\}^{2}$

$Y=9 \alpha V_{0}-4 \mu \omega^{2}-4(E-M) \alpha^{2} V_{0}-2(E-M) \mu \omega^{2}(52)$

\section{A. Non Relativistic Limit}

In this section, we consider the non-relativistic limit of (50). Considering a transformation of the form:

$$
M+E_{n} \rightarrow \frac{2 \mu}{\hbar^{2}} \text { and } M-E_{n} \rightarrow-E_{n},
$$

where $\mu$ is the reduced mass, and substituting it into Eq. (50), we have the non-relativistic energy eigenvalues equation as:

$$
\begin{aligned}
\frac{\hbar^{2}}{2 \mu}\left\{2 n+1\left[\begin{array}{c}
2\left(\alpha^{2} v_{0}+\frac{1}{2} \mu \omega^{2}\right) \\
+2\left(\beta-\alpha v_{0}\right) \frac{2 \mu}{\hbar^{2}}
\end{array}\right]\right\}^{2} \\
E_{n}=\frac{-\frac{\mu^{2} \omega^{2}}{\hbar^{2}}}{9 \alpha v_{0}-4 \mu \omega^{2}-\frac{6 \alpha^{2} v_{0} \mu}{\hbar^{2}}-\frac{4 \mu^{2} \omega^{2}}{\hbar^{2}}}
\end{aligned}
$$

The weight function $\rho(s)$ is obtained from (25) and the parameters of (43) as:

$\rho(s)=s^{2 \sqrt{\frac{1}{4}+\xi_{3}}}$

and using (26) we get the wavefunction $\chi_{n}(s)$ as:

$\chi_{n}(s)=L_{n} \in\left(2 \sqrt{\xi_{1}} s\right)$

where $\epsilon=2 \sqrt{\frac{1}{4}+\xi_{3}}$ and $L_{n} \in\left(2 \sqrt{\xi_{1}} s\right)$ is the Laguerre polynomial. From (28) the wave function is:

$\phi(s)=s^{(1+\epsilon) / 2}$

The radial wave function is then obtained from (30) as:

$R_{n l}(s)=N_{n} s^{\frac{1}{2}+\frac{\epsilon}{2}} L_{n} \in\left(2 \sqrt{\xi_{1}} s\right)$

where $N_{n}$ is the normalization constant.

\section{RESUlTS AND DISCUSSION}

TABLE I: ENERGY LEVEL FOR THE QUANTUM MeChaNICAL PluS

\begin{tabular}{cccc}
\multicolumn{4}{c}{ HARMONIC POTENTIAL } \\
\hline$n$ & $\begin{array}{c}\text { Energy in } \\
(\mathrm{eV})\end{array}$ & $\begin{array}{c}\text { Energy in } \\
(\mathrm{eV})\end{array}$ & $\begin{array}{c}\text { Energy in } \\
(\mathrm{eV})\end{array}$ \\
& For $\alpha=0.2$ & For $\alpha=0.4$ & For $\alpha=0.6$ \\
\hline & & & \\
0 & 2.338516481 & 2.240312424 & 2.456223471 \\
1 & 3.415549442 & 3.520937271 & 3.631148365 \\
2 & 4.492582404 & 4.801562118 & 4.900453713 \\
3 & 5.569615365 & 6.082186966 & 6.143256483 \\
4 & 6.646648326 & 7.362811813 & 7.456723469 \\
\hline
\end{tabular}

We computed numerically the energy spectrum of the quantum mechanical plus harmonic potential using (53) for various values of $n$ with the following parameters of $v_{0}=0.5 \mathrm{fm}^{-1}, \omega=0.5, \mu=h=\beta=1$ for $\alpha=0.2,0.4$ and 
0.6 , respectively as presented in Table I. It is observed that as quantum number is increasing the energy also increases.

\section{CONCLUSION}

In this work, we have obtained the energy eigenvalues and the corresponding un-normalized wavefunction using the parametric NU method for the KGE with the quantum mechanical gravitational and exponential potential plus the harmonic oscillator potential in terms of Laquerre polynomials. We computed the numerical for S- wave and observed that as quantum number increases the energy increases as well.

\section{REFERENCES}

[1] S. Flugge, Practical Quantum Mechanics II, Springer, Berlin, 1971.

[2] C.M. Ekpo, E.P. Inyang, P.O. Okoi, et al., New Generalized MorseLike potential for studying the Atomic interaction in Diatomic Molecules. http://arXiv:2012.02581,2020.

[3] A. N. Ikot, L. E. Akpabio, K. Essien, E. E. Ituen and I. B. Obot, Variational Principle Techniques and the Properties of a cut-off and Anharmonic wave function. E-Journal of Chemistry, Vol. 6, pp. 113119,2009 .

[4] J.E. Ntibi, E.P. Inyang, E. P. Inyang, and E.S. William, Relativistic Treatment of D-Dimensional Klien-Gordon equation with Yukawa potential. International Journal of Innovative Science, Engineering \& Technology Vol. 11(7), pp2348-7968, 2020.

[5] C. Berkdemir, A. Berkdemir and J. Han, Bound state solutions of the Schrödinger equation for modified Kratzer Molecular potential. Chemical Physics Letters.vol.417,pp 326-329,2006.

[6] I.O. Akpan, E.P. Inyang, E.P. Inyang and E.S. William, Approximate solutions of the Schrödinger equation with Hulthen-Hellman potentials for a Quarkonium system. arXiv:2101.01175,2021ю

[7] E.P. Inyang, E.P. Inyang, J.E. Ntibi, and E.S. William, Analytical solutions of Schrodinger equation with Kratzer-screened Coulomb potential for a Quarkonium system. Bulletin of Pure and Applied Sciences, Vol. 40 (D).10.5958/2320-3218.2020.00020.2 (2021) pp. 1424.

[8] C.O. Edet and P.O. Okoi, "Any 1-state solutions of the Schrödinger equation for q-deformed Hulthen plusgeneralized inverse quadratic Yukawa potential in arbitrary dimensions". Revista Mexicana Fisica, 65, pp. 333-344, 2019.

[9] H. Louis, B.I. Ita, O.U. Akakuru, N.A. Nzeata-Ibe, A.I. Ikeuba, T.O. Magu, P.I. Amos and C.O. Edet, 1-state Solutions of the Relativistic and Non-Relativistic Wave Equations for Modified Hylleraas-Hulthen Potential Using the Nikiforov-Uvarov Quantum Formalism, Oriental J. Phys. Sci.3 (2018) 1. http://www.orientjphysicalsciesnces.org/.

[10] E. Omugbe, O.E. Osafile and M.C. Onyeajh, "Mass spectrum of mesons via WKB Approximation method". Advance in High Energy. Phys.10 1143-1155, 2020.

[11] E. P. Inyang, E. S. William and J. A. Obu, Eigensolutions of the Ndimensional Schrödinger equation interacting with Varshni-Hulthen potential model. Revista Mexicana de Fisica. Vol. 67(2), pp. 193-205, 2021.

[12] E.P. Inyang, E. P. Inyang, E.S. William, and E. E. Ibekwe, "Study on the applicability of Varshni potential to predict the mass-spectra of the Quark-Antiquark systems in a non-relativistic framework" Jordan Journal of Physics. Vol. 14(4), pp. 337-345, 2021.

[13] C.O. Edet and P.O. Okoi, "Any 1-state solutions of the Schrödinger equation for q-deformed Hulthen plusgeneralized inverse quadratic Yukawa potential in arbitrary dimensions". Revista Mexicana Fisica, 65, pp. 333-344, 2019.

[14] E.P. Inyang, J. E. Ntibi, E.P. Inyang, E.S. William, and C.C. Ekechukwu, Any L-state solutions of the Schrödinger equation interacting with class of Yukawa-Eckart potentials. International Journal of Innovative Science, Engineering \& Technology, Vol. 11(7), 2432, 2020.

[15] Ikot, A. N., Okorie, U. S., Amadi, P. O., Edet, C. O., Rampho, G. J., \& Sever, R. (2021). The Nikiforov-Uvarov-Functional Analysis (NUFA) Method: A New Approach for Solving Exponential-Type Potentials. Few-Body Systems, 62(1), 1-16. https://doi.org/10.1007/s00601-02101593-5.
[16] E.P. Inyang, E. P. Inyang, E.S. William, E.E. Ibekwe, and I.O. Akpan," Analytical Investigation of Meson Spectrum via Exact Quantization Rule Approach".2020. arXiv:2012.10639.

[17] E.P. Inyang, E.P. Inyang, J.E. Ntibi, E.E., Ibekwe, and E.S. William, "Approximate solutions of D-dimensional Klein-Gordon equation with Yukawa potential via Nikiforov-Uvarov method". Indian Journal of Physics. 2021 https://doi.org/10.1007/s12648-020-01933-X.

[18] E.E. Ibekwe, U.S. Okorie, J.B. Emah, E.P. Inyang and S.A. Ekong, Mass spectrum of heavy quarkonium for screened Kratzer potential (SKP) using series expansion method. Eur. Phys. J. Plus, 87(2021)136. https://doi.org/10.1140/epjp/s13360-021-01090-y.

[19] E. P. Inyang, E. P. Inyang, I. O. Akpan, J. E. Ntibi, E. S. William, Analytical solutions of the Schrödinger equation with class of Yukawa potential for a quarkonium system via series expansion method. EJ. Physics. 2 (2020) 26.http://dx.doi.org/10.24018/ejphysics.2020.2.6.26.

[20] E.P. Inyang, E.P. Inyang, J. Kamiliyus, J.E. Ntibi, \& E.S. William, Diatomic Molecules and Mass Spectrum of Heavy Quarkonium system with Kratzer- screened Coulomb Potential (KSCP) through the solutions of the Schrödinger equation. EJ-Physics 3(2) (2021)55.

[21] C.O. Edet, U.S. Okorie, G. Osobonye, A.N. Ikot, G.J. Rampho and R. Sever, Thermal properties of Deng-Fan-Eckart potential model using Poisson summation approach, J. Math. Chem. 58 (2020) 989. https://doi.org/10.1007/s10910-020-01107-4.

[22] E.P. Inyang, E.P. Inyang, J.E. Ntibi, and E.S. William, Analytical solutions of Schrodinger equation with Kratzer-screened Coulomb potential to the Quarkonium systems (2021) arXiv:2101.01174.

[23] C.O. Edet, U.S. Okorie, G. Osobonye, A.N. Ikot, G.J. Rampho and R. Sever, Thermal properties of Deng-Fan-Eckart potential model using Poisson summation approach, J. Math. Chem. 58 (2020) 989. https://doi.org/10.1007/s10910-020-01107-4.

[24] Edet, C. O., \& Ikot, A. N. (2021). Effects of Topological Defect on the Energy Spectra and Thermo-magnetic Properties of $\$$ CO $\$ \$ C O$ Diatomic Molecule. Journal of Low Temperature Physics, 203, 84-111 (2021). https://doi.org/10.1007/s10909-021-02577-9.

[25] E.P.Inyang ,E.P.Inyang, J.E.Ntibi, E.E.Ibekwe \& E.S. William,Analytical study on the Applicability of Ultra Generalized Exponential Hyperbolic potential to predict the mass spectra of the heavy mesons.arXiv:2101.06389.

[26] Ikot, A. N., Edet, C. O., Okorie, U. S., Abdel-Aty, A. H., Ramantswana, M., Rampho, G. J., Alshehri, N. A., Elagan, S. K.\& Kaya, S. (2021). Solutions of the 2D Schrodinger equation and its thermal properties for Improved Ultra Generalized Exponential Hyperbolic potential (IUGEHP). Eur. Phys. J. Plus 136(2021) 434 https://doi.org/10.1140/epjp/s13360-021-01408-w.

[27] E. P. Inyang, J. E. Ntibi, E.A. Ibanga, F. Ayedun, E. P. Inyang, E.E. Ibekwe, E. S. William \& I.O. Akpan. "Thermodynamic properties and mass spectra of a quarkonium system with Ultra Generalized Exponential- Hyperbolic potential". Communication in Physical Science, 2021, 7(2), pp. 97-114.

[28] I.O. Akpan, E.P. Inyang, E.P. Inyang and E.S. William, Approximate solutions of the Schrödinger equation with Hulthen-Hellman potentials for a Quarkonium system. Rev.Max.Fis. 67(3), $482-490$ (2021).https://doi.org/10.31349/RevMaxFis.67.482.

[29] Ikot, A. N., Edet, C. O., Okorie, U. S., Abdel-Aty, A. H., Ramantswana, M., Rampho, G. J., Alshehri, N. A., Elagan, S. K.\& Kaya, S. (2021). Solutions of the 2D Schrodinger equation and its thermal properties for Improved Ultra Generalized Exponential Hyperbolic potential (IUGEHP). Eur. Phys. J. Plus 136(2021) 434 https://doi.org/10.1140/epjp/s13360-021-01408-w.

[30] K. J. Oyewumi and K. D. Sen, Exact solutions of the Schrödinger equation for the pseudoharmonic potential:an application to some diatomic molecules. Journal of Mathematical Chemistry. vol. 50, pp. 1039-1059, 2012.

[31] E.S. William, J.A. Obu, I.O. Akpan, E.A. Thompson, and E.P. Inyang, Analytical Investigation of the Single-particle energy spectrum in Magic Nuclei of $56 \mathrm{Ni}$ and $116 \mathrm{Sn}$. European Journal of Applied Physics. vol. 2, 28, 2020

[32] Wen, X. J., Zhong, X. H., Peng, G.X., Shen, P.N. \& Ning, P. Z. (2005) Thermodynamics with density and temperature dependent particle masses and properties of bulk strange quark matter and strangelets. Chinese Physics Letters, 26, 235-246.

[33] Ita B.I., Ikeuba, A.I and Ikot, A.N Solutions of the Schrodinger equation with quantum mechanical plus harmonic oscillator potential. Commun. Theor. Phys. 61:149-152.

[34] K. J. Oyewumi and K. D. Sen, Exact solutions of the Schrödinger equation for the pseudoharmonic potential:an application to some diatomic molecules. Journal of Mathematical Chemistry. vol. 50, pp. 1039-1059, 2012.

[35] E.S. William, E.P. Inyang, and E.A. Thompson, "Arbitrary $\ell$-solutions of the Schrödinger equation interacting with Hulthén-Hellmann 
potential model”. Revista Mexicana de Fisica. Vol. 66(6), pp. 730-741, Nov. 2020.

[36] Edet, C. (2020). Effects of Magnetic and Aharanov-Bohm (AB) Fields on the Energy Spectra of the Yukawa Potential. arXiv preprint arXiv:2012.08644.

[37] Ikot A.N, Antia A.D, Akpabio L. E and Obu J. A, Analytical solutions of Schrodinger equation with Two-dimensional harmonic potential in Cartesian and polar coordinates via Nikiforov-Uvarov method. Journal of Vectorial Relativity 6(2), 65-76, 2011.

[38] A. Arda and R.Sever, Exact solutions of Schrödinger equation via Laplace transform approach:pseudoharmonic potential and Mie-type potentials. Journal of Mathematical Chemistry. vol. 50, pp. 971-980, 2012.

[39] A. F. Nikiforov and V. B. Uvarov, Special Functions of Mathematical Physics (Birkhauser: Bassel)1988.

[40] A. N. Ikot, L. E. Akpabio, K. Essien, E. E. Ituen and I. B. Obot, Variational Principle Techniques and the Properties of a cut-off and Anharmonic wave function. E-Journal of Chemistry, Vol. 6, pp. 113$119,2009$.

[41] S. W. Qian, B. W. Huang \& Z.Y. Gu., Supersymmetry and shape invariance of the effective screened potential. New Journal of Physics, 4, 13-19, 2002.

[42] W.C. Qiang \& S. H. Dong, Analytical approximations to the solutions of the Hulthen potential with centrifugal term. Physics Letters A, 368, 13-17. 2007.

[43] Ikhdair S M and Sever R 2008Int. J. Mod. Phys.191425 S. M. Ikhdair and R. Sever, Exact quantization rule to the Kratzer- type potentials: an application to the diatomic molecules. Journal of Mathematical Chemistry, vol. 45, pp. 1137-2009, 2009.

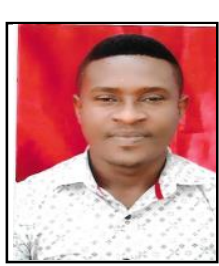

E.P. Inyang was born in Calabar, Cross River state on 26 November. He holds PhD in Theoretical Physics, M.Sc. in Nuclear Physics, PGD in Physics and B.Sc. Education Physics all from University of Calabar, Calabar, Cross River State, Nigeria. He has over10 years of teaching experience both in Post Primary and Post Secondary schools.

$\mathrm{He}$ is currently a Lecturer in the Department of Physics, National Open University of Nigeria, Abuja. He has published in reputable journals of Physics:

1. E. P. Inyang, E. P. Inyang, E. S. William, and E. E. Ibekwe, "Study on the applicability of Varshni potential to predict the mass-spectra of the Quark-antiquark systems in a non-relativistic framework", Jordan Journal of Physics, Vol.14(4),pp337-345, 2021.

2. E. P. Inyang, E. P. Inyang, and M. B. Latif, "A Correlation: TL response of synthetic fused quartz with 60Co gamma (high dose) source and $90 \mathrm{Sr} / 90 \mathrm{Y}$ Beta (low dose) source", Bulletin of Pure and applied Sciences-Physics, vol. 38(1), pp. 6-12, 2019.

3. E. P. Inyang, E. S. William and J. A. Obu, "Eigen solutions of the $\mathrm{N}$-dimensional Schrödinger equation interacting with Varshni-Hulthen potential" Revista Mexicana de Fisica. 67, 2021.

4. E.E. Ibekwe, U.S. Okorie, J.B. Emah, E.P. Inyang and S.A. Ekong, Mass spectrum of heavy quarkonium for screened Kratzer potential (SKP) using series expansion method. European Physical Journal Plus 87 (2021), 136.

Dr. Inyang is currently researching on high energy Physics and he is a member of Nigerian Institute of Physics. 